\title{
Une approche multidimensionnelle de l'optimisation de la radioprotection
}

\author{
A. OUDIZ (*) \\ (Reçu le 16 novembre 1979)
}

\section{RÉSUMÉ}

Le principe d'optimisation de la radioprotection est souvent associé à la nécessité de prendre en compte la dimension collective du risque sanitaire. En réalité l'optimisation et l'approche collective de la radioprotection ne devraient pas être confondues. Il est en effet possible d'aborder l'optimisation en intégrant les multiples dimensions du détriment sanitaire.

A titre d'illustration une méthode d'optimisation multicritère a été mise en œuvre dans le contexte de la détermination des niveaux "ALARA " pour le cycle du combustible PWR. Une telle méthode a permis de tenir compte simultanément du risque sanitaire et du risque associé aux groupes critiques. Les résultats obtenus montrent que l'optimisation ainsi conçue permet de réaliser une synthèse satisfaisante de l'approche traditionnelle dite irdividuelle et de l'approche collective.

De façon générale, la crédibilité de la procédure d'optimisation dépendra pour une bonne part de la manière dont on aura su tenir compte de la multidimensionnalité inhérente à la plupart des choix de radioprotection.

\section{ABSTRACT}

The optimization principle as applied to radiation protection is often associated with the necessity to take into account the collective dimension of the health risk. Actually, there should be no confusion between optimization and the collective approach of radiation protection. It is possible to consider optimization in such a way that most of the various dimensions of the health detriment are included. In order to illustrate this an optimization method based on multiattribute analysis was implemented within the framework of the determination of "ALARA" levels for the PWR fuel cycle. With this method both the collective health risk and the risk related to the critical group are simultaneously taken into account. The results show that such an optimization leads to a satisfactory synthesis of the conventional individual approach and the collective approach.

More generally, the credibility of the optimization process will largely rely upon the ability to take into account the multiple dimensions of most of the choices in the field of radiation protection.

(*) Association Euratom/C.E.A.-DPr, Centre d'Études nucléaires de Fontenay-aux-Roses B. P. $n^{\circ} 6,92260$ Fontenay-aux-Roses. 
Les recommandations récentes de la Commission internationale de Protection radiologique [1] relatives à l'optimisation de la protection entérinent l'évolution de la doctrine dans deux domaines qu'il convient de distinguer clairement. Tout d'abord, il apparaît désormais nécessaire de prendre en compte la dimension collective du risque radiologique. D'autre part, il faudra à l'avenir gérer de façon plus « rationnelle » le risque radiologique, à l'aide de méthodes d'aide à la décision parmi lesquelles la C.I.P.R. privilégie, de manière peut-être excessive, l'analyse coût-avantage. Les praticiens chargés de la mise en œuvre de ces nouveaux principes tendent actuellement à les interpréter de façon erronnée en confondant l'approche collective et la procédure d'optimisation. La procédure d'optimisation ne consiste pas à remplacer l'approche individuelle (groupes critiques) par l'approche collective puis à vérifier que le résultat de l'optimisation est bien en deçà des " garde-fous » que représentent les limites de dose.

Il paraît clair que les études d'optimisation n'auront de crédibilité que dans la mesure où elles seront conçues de manière à prendre en compte simultanément les diverses dimensions du risque radiologique. A cet égard, l'évolution de la doctrine de la C.I.P.R. ne fait qu'entériner la nécessité de se doter à l'avenir d'indicateurs du risque radiologique moins restrictifs que par le passé. En particulier, au risque individuel subi par les populations les plus exposées (groupes critiques), il faut désormais ajouter, et non substituer, un indicateur du risque sanitaire collectif. Cette adjonction résulte, logiquement, en effet, de l'hypothèse communément admise de linéarité sans seuil des relations dose-risque aux faibles doses. L'équivalent de dose collectif ou ses formes les plus élaborées (engagement d'équivalent de dose collectif...) constitue dès lors un indicateur commode du risque sanitaire collectif.

Pour être utilisée de façon réaliste et par conséquent crédible, la méthode d'optimisation par analyse coût-avantage, présentée par la C.I.P.R., devrait tenir compte explicitement des deux dimensions du risque radiologique évoquées plus haut. De ce fait, la mise en œuvre de cette méthode serait, en réalité, plus complexe que ne le suggèrent les publications 22 et 26 de la C.I.P.R. Une telle complexité n'entraîne certes pas l'impossibilité de recourir à l'analyse coût-avantage, mais la bidimensionnalité du risque radiologique et, en réalité, sa multidimensionnalité incitent à s'orienter vers des méthodes d'aide à la décision qui s'accomodent mieux d'un tel caractère.

C'est ainsi que nous avons été conduits à explorer les possibilités qu'offre une analyse multicritère dans le problème d'optimisation suivant : Recherche des niveaux d'équivalent de dose "ALARA » $\left({ }^{1}\right)$ liés aux activités du cycle du combustible des réacteurs à eau pressurisée (PWR) en ce qui concerne le public [2]. Dans cette étude à caractère strictement méthodologique, nous nous sommes appuyés sur une base de données fournie par des rapports de l'E.P.A. des États-Unis [3,4]. Nous disposions d'environ une quarantaine d'options de traitement des effluents liquides et gazeux relatives aux diverses

(1) «As low as reasonably achievable », en français : « les plus faibles que l'on puisse raisonnablement atteindre " (C.I.P.R.). 
installations du cycle du combustible. La recherche des niveaux d'équivalent de dose "Alara " passait par la sélection des options de traitement devant conduire à de tels niveaux. Chaque option a été caractérisée par son coût, par la dose individuelle au groupe critique qu'elle permettrait d'éviter, par le risque sanitaire collectif public évité $\left({ }^{2}\right)$ ainsi que par une note traduisant la fiabilité des données technico-économiques tirées des rapports de l'E.P.A.

Dans ce problème décisionnel interviennent, en réalité, d'autres dimensions du risque telles, par exemple, que le risque subi par les travailleurs du fait du choix de telle option de traitement des effluents, ou bien telles que le risque sanitaire lié au fonctionnement perturbé des installations. Bien que le problème posé ait trait au choix d'options de traitement destinées à la protection du public durant le fonctionnement normal des installations, les dimensions précédentes doivent être introduites dans la mesure où elles sont prises en compte dans les pratiques décisionnelles de protection. Le manque de données ne nous a pas permis d'intégrer ces deux dimensions dans notre étude, mais la note de fiabilité technico-économique, au-delà de la définition qu'on lui a attribué, atteste de la capacité de la méthode à prendre en compte des critères de choix multiples.

Dans la sélection des options de traitement conduisant aux niveaux " ALARA ", nous avons été confrontés, cela est inévitable, quelle que soit la méthode employée, au problème de la pondération des critères. En particulier, nous avons été amenés à affecter un poids relatif au risque individuel, au risque collectif, au coût.

C'est à ce stade que nous rencontrons le problème controversé de la valeur de la vie humaine, en fait, dans ce cas, de la valeur de l'effet sanitaire évité. Mais, contrairement à ce qui se passe dans l'analyse coût-avantage, dans laquelle la détermination de cette valeur par des procédures plus ou moins scientifiques constitue la clef de voûte de l'étude, la valeur retenue apparaît ici comme un paramètre, important certes, mais non crucial, comme le prouvent les analyses de sensibilité. Ce paramètre reflète, au même titre que celui qui relie le risque individuel au risque collectif, les choix des décideurs quant au montant des ressources financières qu'il convient d'affecter pour éviter un effet sanitaire, dans les groupes critiques ou bien dans la population en général. Dans ces choix, l'analyste chargé de conduire l'étude ne peut se substituer aux décideurs.

A l'analyste revient la tâche d'éclairer les décideurs sur l'importance que revêt le choix des valeurs adoptées pour chacun des paramètres, à travers des analyses de sensibilité. Dans l'étude, nous avons adopté arbitrairement une valeur évoquée par l'E.P.A. : 150000 \$ pour l'effet sanitaire évité dans la population générale. Quant à la valeur de l'effet sanitaire évité dans les groupes critiques, nous avons considéré - à titre d'exemple - que l'on pouvait dépenser dix fois plus pour éviter l'apparition d'un effet sanitaire supplémentaire, ceci en vertu de considérations d'ordre éthique liées au fait

$\left({ }^{2}\right)$ Égal au produit de l'équivalent de dose collectif par le coefficient dose-risque correspondant à l'organe considéré.

vol. $15-\mathrm{N}^{\circ} 1$ 
que les groupes critiques constituent des groupes à plus haut risque, identifiés a priori.

De tels choix, encore une fois arbitraires et susceptibles de modification, nous ont conduit à la sélection d'une vingtaine d'options parmi les quarante disponibles à l'origine. La selection a été effectuée en recourant à une procédure de classification inspirée d'une méthode multicritère, dite " Electre I ". Les options retenues confirment les choix de radioprotection déjà effectués dans le passé à l'aide de l'approche "individuelle », basée sur les groupes critiques, et les options nouvelles portent sur les radionucléides tels que les gaz rares radioactifs, le tritium, l'iode 129 , ceci n'est évidemment pas surprenant. Il apparaît ainsi, et c'est une des conclusions importantes de l'étude, que l'optimisation n'a pas remis en cause les choix antérieurement effectués, mais qu'elle les a complétés en mettant en avant les options nouvelles qui retiendront à l'avenir l'attention des responsables confrontés à l'extension mondiale des programmes électronucléaires.

L'approche multicritère confirme donc ainsi son caractère synthétique. Par ailleurs, les analyses de sensibilité montrent que la valeur implicite de l'effet évité pourrait varier entre 100000 et $500000 \$$ sans que pour autant, les choix soient modifiés. Elles confirment, de plus, que l'étude aurait grandement gagné en crédibilité si nous avions pu introduire un indicateur du comportement des options de traitement des effluents en cas de fonctionnement perturbé. Ceci est particulièrement vrai pour le piégeage des iodes dans les réacteurs, dont la justification ne s'impose pas toujours dans les analyses de sensibilité du fait même que nous n'avons pu rendre compte de l'intérêt qu'elles présentent en cas de fonctionnement perturbé.

La démarche d'optimisation, classique dans certains domaines, constitue une innovation dans le contexte de la radioprotection. Pour qu'elle y apparaisse comme un facteur de progrès, il lui faudra démontrer, en particulier, son aptitude à prendre en compte la multidimensionnalité inhérente à la plupart des choix de protection. De cette aptitude, dépendra pour une bonne part sa crédibilité auprès de ceux qui ont une expérience concrète de la radioprotection.

\section{BIBLIOGRAPHIE}

[1] International Commission on Radiological Protection, Recommendations... of the I.C.R.P. Publication 26, Oxford, Pergamon Press, 1977.

[2] Lombard J., OUdiz A., Optimisation des rejets de la filière PWR par une méthode multicritère, Rapport Euratom-C.E.A. (à paraître).

[3] U.S. Environmental Protection Agency, Environmental radiation protection requirements for normal operations of activities in the fuel cycle (40-CFR 190) Final Environmental Statement. EPA-520/4-76-016, 1976.

[4] U.S. Environmental Protection Agency, Environmental analysis of the uranium fuel cycle, 4 vol., EPA-520/9-73-003-B, C et D, 1973 et EPA-520/4-76-017, 1976. 